Check for updates

Cite this: Chem. Commun., 2019, 55, 10253

Received 24th June 2019,

Accepted 2nd August 2019

DOI: $10.1039 / \mathrm{c} 9 \mathrm{cc} 04846 \mathrm{~d}$

rsc.li/chemcomm

\section{Phenolic hydrogen bond donors in the formation of non-ionic deep eutectic solvents: the quest for type V DES $\dagger$}

\author{
Dinis O. Abranches, (D) ${ }^{a}$ Mónia A. R. Martins, (D) ${ }^{a b c}$ Liliana P. Silva, (D) \\ Nicolas Schaeffer, (D) ${ }^{\text {a }}$ Simão P. Pinho (D) ${ }^{\text {bc }}$ and João A. P. Coutinho (D) *a
}

\begin{abstract}
Mixtures of non-ionic compounds have been reported as DES but most are just ideal mixtures. In the thymol-menthol system, an abnormal strong interaction was identified stemming from the acidity difference of the phenolic and aliphatic hydroxyl groups. This type of interaction is found to be the key to prepare non-ionic DES, that may be classified as type $\mathrm{V}$.
\end{abstract}

Eutectic solvents are, for any given temperature, liquid mixtures of weak Lewis or Brønsted acids and bases that, when pure, are solid at that temperature. In 2003, Abbot et al. ${ }^{1}$ reported that binary mixtures of urea and several solid quaternary ammonium salts were liquid at room temperature and presented interesting solvent properties. These liquid mixtures were later named deep eutectic solvents (DES). ${ }^{2}$ The prefix deep served to highlight a eutectic temperature much lower than the melting temperature of the pure components. However, since the resulting eutectic temperature of a mixture is not only governed by the specific interactions established between its components but also by their melting properties, the term deep should preferably be used to emphasize a eutectic temperature much lower than that predicted by assuming a thermodynamic ideal behaviour of the liquid phase, ${ }^{3}$ resulting from stronger interactions between the DES precursors than those present in the pure compounds. ${ }^{4}$

In the last decade, a large amount of research on DES has been carried, from fundamental studies to applications. ${ }^{4-9}$ The binary nature of DES allows for the formulation of a tailored solvent for a given application through judicious selection of its constituents. However, most DES are based on at least one ionic species and dominated by strong hydrogen bonding

\footnotetext{
${ }^{a}$ CICECO - Aveiro Institute of Materials, Department of Chemistry, University of Aveiro, 3810-193 Aveiro, Portugal. E-mail: jcoutinho@ua.pt

${ }^{b}$ Associate Laboratory LSRE-LCM, Department of Chemical and Biological Technology, Polytechnic Institute of Bragança, 5300-253 Bragança, Portugal ${ }^{c}$ Mountain Research Center - CIMO, Polytechnic Institute of Bragança,

5301-855 Bragança, Portugal

$\dagger$ Electronic supplementary information (ESI) available: Experimental procedure; thermodynamic and computational details; pertinent chemical structures and literature data. See DOI: 10.1039/c9cc04846d
}

interactions, rendering the resulting mixture hydrophilic. The majority of the DES reported to date are classified into four main types depending on their constituents: ${ }^{4}$

Type I - quaternary ammonium salt + metal chloride;

Type II - quaternary ammonium salt + metal chloride hydrate;

Type III - quaternary ammonium salt + hydrogen bond donor;

Type IV - metal chloride hydrate + hydrogen bond donor.

The design of hydrophobic eutectic mixtures solely composed of non-ionic species has received particular recent attention in the literature. Such molecular eutectics overcome the issues associated with hydrophobic eutectics based on large quaternary ammonium salts, namely their higher cost, hydrophobicity and viscosity. ${ }^{10}$ Furthermore as the proposed DES are based on non-ionic species, this allows for the potential recovery and regeneration of the eutectic by evaporation, a significant advantage over ionic DES. Martins et $a l .{ }^{11}$ reported the solid-liquid phase diagram of binary systems composed of thymol or menthol and several carboxylic acids, but found all those systems to be ideal mixtures and, thus, not true deep eutectic solvents. The solid-liquid phase diagram for the mixtures of ibuprofen with thymol or menthol, ${ }^{12}$ and of menthol with testosterone, ${ }^{13}$ were reported but these mixtures also behave ideally. ${ }^{3}$ On the other hand, Lazerges et al. ${ }^{14}$ showed that the thymol/lidocaine system is liquid at room temperature, in a large composition range, due to negative deviations from ideality presented by both components. As such, this system is a true deep eutectic solvent.

In contrast with the examples above, most studies on nonionic DES focus on investigating the melting temperature of specific stoichiometric mixtures of substances and do not study their phase behaviour. This hampers any conclusions about the deep character of the resulting system, even when liquid at room temperature. Besides, it prevents the identification of patterns and specific interactions that may originate new DES. For instance, van Osch et al. ${ }^{15}$ prepared 507 combinations of non-ionic natural substances and identified 17 liquid mixtures, 8 of which based on thymol. However, it is not clear from their 


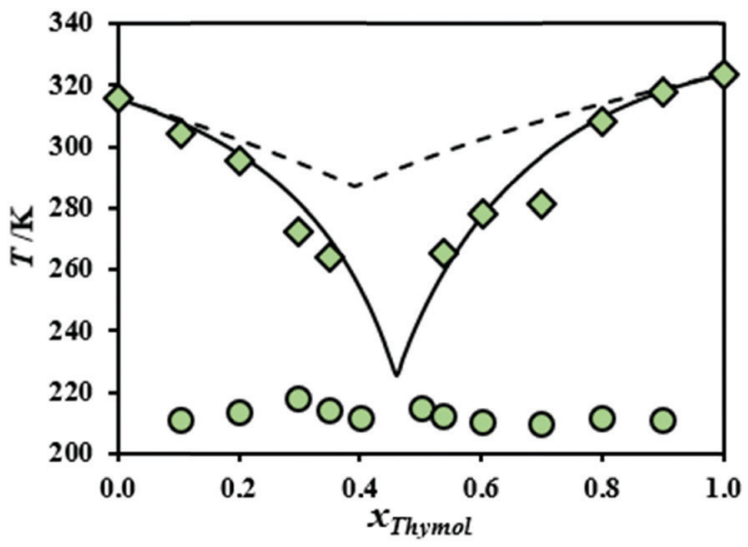

Fig. 1 Experimental solid-liquid equilibrium phase diagram for the system thymol/menthol $(\Delta)$, measured using DSC. The predictions by the ideal liquid phase model (dashed line) and by COSMO-RS (full line) are included. Glass transition temperatures are also shown (O). Activity coefficients displayed in Fig. S2 (ESI†).

work whether the melting temperature depression observed in the studied systems is due to specific, strong interactions between their components or simply due to their melting properties, as in the case of the thymol systems investigated by Martins et al. ${ }^{11}$

By measuring the solid-liquid equilibrium phase diagram of the system thymol/(-)-menthol (henceforth referred to as menthol), reported in Fig. 1 and Table S1 (ESI $\dagger$ ), a new true deep eutectic solvent was here identified. This is quite remarkable since, even though both substances are structurally very similar (Fig. S1, ESI $\dagger$ ), they present severe negative deviations to ideality when mixed. It has been known for more than a century, ${ }^{16}$ though, that thymol forms a liquid, when mixed with other terpenes. Moreover, recently some authors proposed that a mixture with a 1:2 mole ratio of thymol:menthol was a DES. ${ }^{15}$ However, since the phase behaviour of the mixture was not investigated, the unique behaviour of the system was not recognised, nor the nature or stoichiometry of its interactions.

The COSMO-RS model ${ }^{17,18}$ is shown in Fig. 1 to be able to correctly describe the non-ideality of the thymol/menthol system. This predictive model relies on pair-wise interactions between each molecule type in a mixture in order to evaluate the resulting activity coefficients. For this particular case, the geometry of the strongest pair-wise interaction for each possible combination (menthol/menthol, thymol/thymol and thymol/menthol), considered by COSMO-RS in its calculations, was optimised. These results are reported in Fig. 2 and clearly show that the non-ideality of the system results from a hydrogen bond established between thymol and menthol that is much stronger that the hydrogen bonding present in any of the pure substances. Even though the phase behaviour reported in Fig. 1 was not anticipated due to the structural similarity of the substances and their ability to establish hydrogen bonds in their pure phases, COSMO-RS provides important clues to understand the interactions on this system.

The severe negative deviations from ideality of the thymol/ menthol system may be interpreted through resonance effects.
Fig. 3 depicts three resonance structures of thymol. Even though the first structure is more stable than its counterparts (the true chemical structure of thymol possesses contributions from all of its resonance structures, resulting in an oxygen with $\mathrm{sp}^{2}$ character), the lone electron pairs of the oxygen in thymol are delocalized through the aromatic ring, which makes its hydroxyl group more positive than usual. As such, the oxygen becomes a worse hydrogen bond acceptor whilst its bonded proton becomes a better hydrogen bond donor. Thus, in its pure liquid phase, thymol establishes hydrogen bonds between a "good" hydrogen bond donor and a "poor" hydrogen bond acceptor. On the other hand, since no aromaticity is present, menthol possesses a normal hydroxyl group, forming regular hydrogen bonds in its pure liquid phase. When thymol and menthol are mixed, a preferable interaction is established, where the hydrogen of thymol (a better than usual hydrogen bond donor) interacts favourably with the oxygen of menthol, forming a hydrogen bond that is stronger than any present in the pure liquid components. In turn, this leads to the severe negative deviation from ideality presented by both thymol and menthol in Fig. 1 .

The above assumption is further supported by the thermodynamic behaviour of the systems phenol/cyclohexanol and benzyl alcohol/cyclohexanol (the activity coefficients for these systems, obtained from vapor-liquid equilibrium data, are depicted in Fig. S3, ESI $\dagger$ ). Notice that the structures of phenol and cyclohexanol are simpler versions of the structures of thymol and menthol, without the side alkyl chains. When mixed, phenol and cyclohexanol present severe negative deviations from ideality, analogous to the system thymol/menthol. This demonstrates that these deviations are not specific to thymol but to the hydroxyl group connected to the aromatic ring. On the other hand, since the hydroxyl group of benzyl alcohol is not directly attached to its aromatic ring, which prevents the resonance effects previously discussed, a drastic change in behaviour is observed. Both benzyl alcohol and cyclohexanol present positive deviations from ideality when mixed, the opposite of the phenol/cyclohexanol system, meaning that both substances establish stronger interactions in their pure phases than when mixed.

The specific interaction identified on the system thymol/ menthol is not particular to that system (as illustrated with the phenol/cyclohexanol example). The results here reported show that a hydroxyl group directly attached to an aromatic ring, i.e. a phenolic compound, may form a DES when mixed with a hydrogen bond acceptor, even if that acceptor is able to establish hydrogen bonds with itself. This allows for the design of novel non-ionic DES, as discussed below. Deep eutectic solvents based on this kind of hydrogen bond should thus be considered as a new type of DES: type $\mathrm{V}$, composed only of molecular substances.

Since COSMO-RS proved to be able to describe the thymol (THY)/menthol (MEN) system, this tool is now exploited to predict the solid-liquid equilibrium phase diagram of new mixtures. The six compounds discussed (see Fig. S4 for their structures, ESI $\dagger$ ), coumarin (COU), R-camphor (CAM), hydroquinone (HYD), butylated hydroxytoluene (BHT), 4-nitrophenol (4NP) and salicylic acid (SAL) 

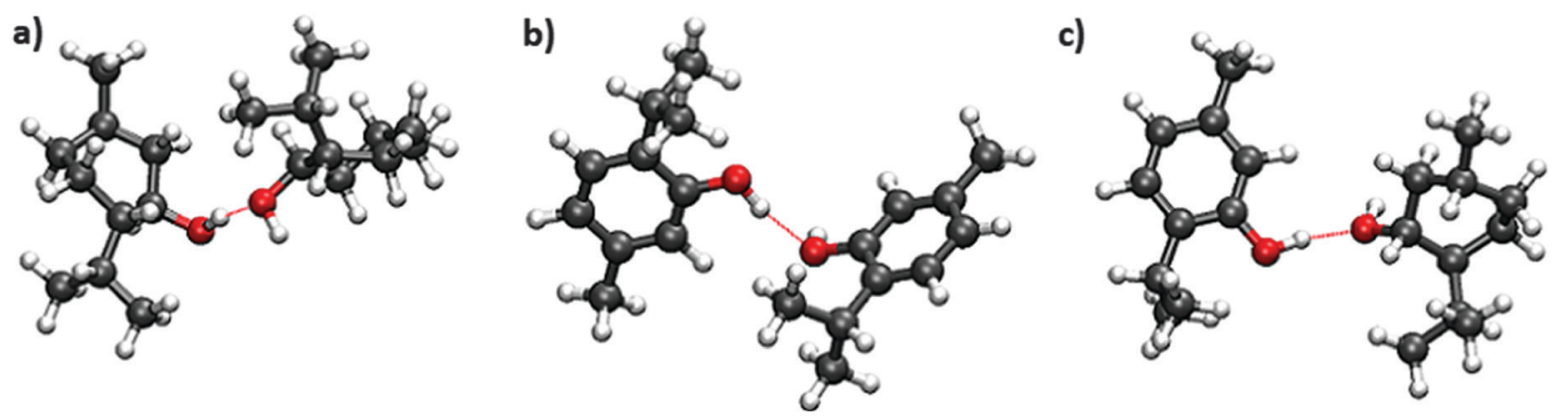

Fig. 2 Optimized geometries (COSMO solvation model) of the interaction pairs recognised by COSMO-RS as most probable to be found in (a) pure liquid menthol, (b) pure liquid thymol and (c) liquid thymol/menthol mixture. The interaction energy and the hydrogen bond length (red dotted line) are, respectively, (a) $9.6 \mathrm{~kJ} \mathrm{~mol}^{-1}$ and $0.185 \mathrm{~nm}$, (b) $0.5 \mathrm{~kJ} \mathrm{~mol}^{-1}$ and $0.187 \mathrm{~nm}$ and (c) $14.8 \mathrm{~kJ} \mathrm{~mol}^{-1}$ and $0.171 \mathrm{~nm}$.
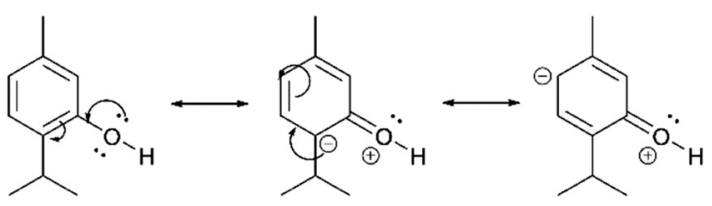

Fig. 3 Resonance structures of thymol, evidencing the $\mathrm{sp}^{2}$ nature of its oxygen atom.

were selected to understand which type of phenolic molecules can favourably establish strong hydrogen bonds with hydrogen bond acceptors and, thus, form type V DES. This includes studying the presence of electron withdrawal or donning moieties around the phenolic ring and the number and type of hydrogen bond interaction sites. The predicted solid-liquid phase diagrams are reported in Fig. S5 (ESI $\dagger$ ) whilst the corresponding activity coefficients are depicted in Fig. 4. For each system, a mixture was prepared with a composition close to the predicted eutectic point and its melting temperature was measured using DSC (see ESI $\dagger$ ). The comparison, reported in Table 1, between each mixture experimental behaviour and the eutectic temperature predicted by the ideal liquid model indicates the deepness of the eutectic solvent. Two additional hydrogen bond acceptors are used (coumarin and R-camphor) to reinforce that menthol has no exclusive role in the interaction explained above. This is clearly demonstrated by the system thymol/coumarin.

While phenol and thymol possess hydroxyl groups with a positive character, this does not seem to be the case for hydroquinone. COSMO-RS predicts an almost ideal behaviour for it when mixed with menthol or camphor and it is experimentally shown that the system possesses positive deviations to ideality (Table 1). Since hydroquinone possesses an additional hydroxyl group, both act as resonance electron donors to the other, attenuating their otherwise positive character. In other words, the second hydroxyl group acts as an activating group for the aromatic ring.

In line with the last paragraph, 4-nitrophenol acts very differently from hydroquinone. Contrary to the hydroxyl group, the nitro group is known as a deactivating group, meaning it is a resonance electron withdrawer. Thus, the presence of the nitro group in the aromatic ring further increases the positive character of the hydroxyl group. This effect competes, though, with the fact that the nitro group acts as a hydrogen bond acceptor, which allows for stronger interactions in its pure phase than phenol or thymol. However, as Fig. 4 predicts and Table 1 proves, the 4-nitrophenol-based systems are type $\mathrm{V}$ deep eutectic solvents.

Butylated hydroxytoluene (BHT) would be an advantageous phenolic compound to use in the production of DES due to its low toxicity, low market price and applicability as an antioxidant. However, the high steric hindrance present around its hydroxyl group prevents the formation of type V DES.

Finally, Fig. 4 and Table 1 give important insight into the use of aromatic carboxylic acids to prepare DES of type V. When mixed with menthol or coumarin, salicylic acid presents negative deviations from ideality. This is remarkable since carboxylic acids typically form ideal mixtures with hydrogen bond acceptors, as exemplified by Martins et al. ${ }^{11}$ Carboxylic acids are both good hydrogen bond donors and acceptors, being able to form very strong hydrogen bonding in their pure phase and even stable dimmer complexes. As such, in most mixtures they do not significantly interact with the other components, leading to a thermodynamic ideal behaviour or even positive deviations to it.

The phenolic hydrogen bond studied in this work using the thymol/menthol system as model may thus be the basis for the development of new deep eutectic solvents of type V. With the aid of COSMO-RS it was shown that several phenolic compounds may be used to prepare this new type of DES. The major criteria for a successful DES-forming phenolic compound is that it should not possess good hydrogen bond acceptor centres nor strong activating groups in the aromatic ring. The second component needs only to be a hydrogen bond acceptor. It is here suggested that ketones and amines may even work better than hydroxyl groups at forming new DES of type $\mathrm{V}$, since they usually cannot establish hydrogen bonds in their pure phases.

Deep eutectic solvents have been extensively investigated as a green extraction media for natural phenolic compounds. ${ }^{9}$ Among other applications, phenolic-based type V DES could prove particularly useful in this regard, since its phenolic component could boost the solubility of other phenolic compounds or any other aromatic compounds through the formation 

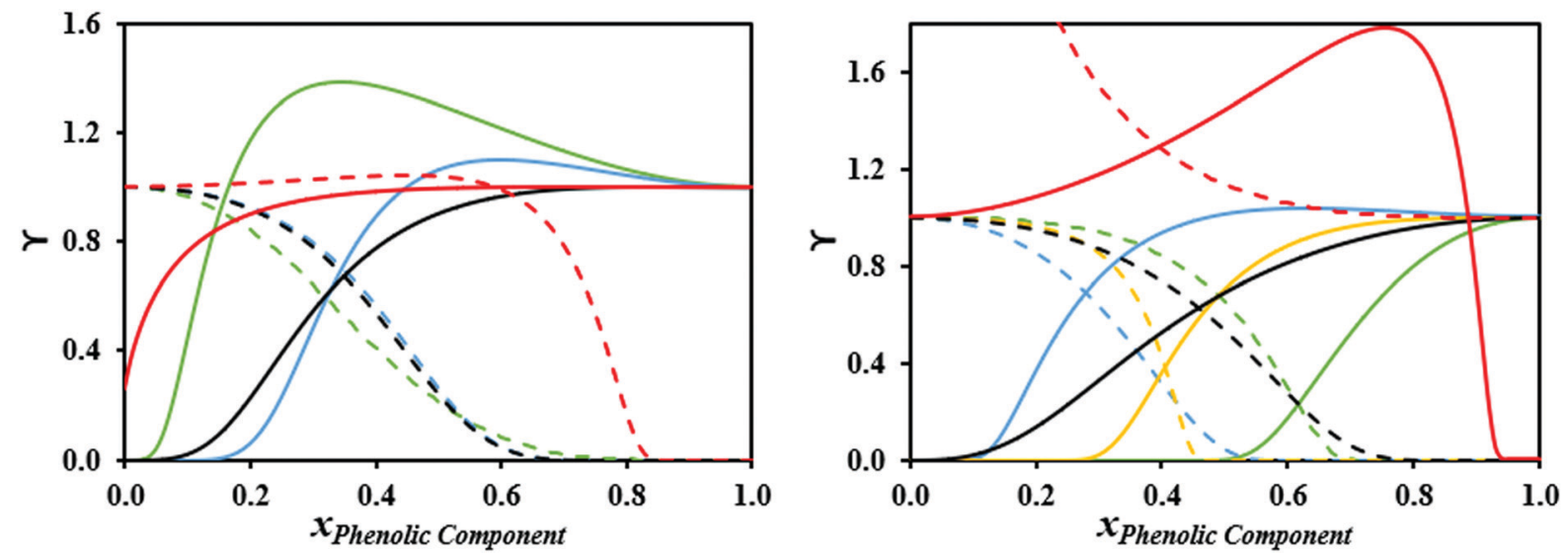

Fig. 4 Non-isothermal activity coefficients of potential type $V$ DES, estimated by COSMO-RS whilst calculating their solid-liquid equilibrium diagram. Full lines represent the phenolic component while dashed lines represent the second component. Left panel: Hydroquinone + menthol (green), 4-nitrophenol + menthol (blue), salicylic acid + menthol (black) and BHT + menthol (red). Right panel: Thymol + coumarin (green), hydroquinone + camphor (blue), 4-nitrophenol + camphor (yellow), salicylic acid + coumarin (black) and BHT + coumarin (red).

Table 1 Experimental melting temperature $\left(T_{m}\right)$ of potential type $V$ DES, along with predictions by the ideal liquid model and by COSMO-RS

\begin{tabular}{|c|c|c|c|}
\hline System $\left(\mathrm{mol} \mathrm{mol}{ }^{-1}\right)$ & Ideal $T_{\mathrm{m}} / \mathrm{K}$ & COSMO-RS $T_{\mathrm{m}} / \mathrm{K}$ & Exp. $T_{\mathrm{m}} / \mathrm{K}$ \\
\hline THY $(0.65)+\operatorname{COU}(0.35)$ & 305.5 & 269.1 & $290.9^{a}$ \\
\hline $\operatorname{HYD}(0.2)+\operatorname{MEN}(0.8)$ & 365.2 & 371.6 & 398.5 \\
\hline HYD $(0.25)+$ CAM $(0.75)$ & 374.5 & 353.2 & 397.5 \\
\hline $4 \mathrm{NP}(0.3)+\mathrm{MEN}(0.7)$ & 319.2 & 288.5 & 266.1 \\
\hline $4 \mathrm{NP}(0.4)+\mathrm{CAM}(0.6)$ & 333.2 & 289.0 & 281.5 \\
\hline SAL $(0.15)+$ MEN $(0.85)$ & 333.7 & 303.1 & 297.9 \\
\hline $\operatorname{SAL}(0.3)+\operatorname{COU}(0.7)$ & 364.1 & 318.3 & 338.9 \\
\hline BHT (0.4) + MEN (0.6) & 302.1 & 301.5 & $299.2^{b}$ \\
\hline BHT $(0.5)+\mathrm{COU}(0.5)$ & 310.9 & 325.9 & 322.1 \\
\hline
\end{tabular}

${ }^{a}$ Eutectic temperature below $273 \mathrm{~K} .{ }^{b}$ Eutectic temperature is $295.5 \mathrm{~K}$.

of $\pi$-interactions between solvent and solute. Alternatively, phenolic substances could provide good solvents for the extraction of some types of biomolecules with hydrogen bond acceptors by in situ formation of DES.

This work was developed in the scope of the project CICECO Aveiro Institute of Materials, POCI-01-0145-FEDER-007679 (Ref. FCT UID/CTM/50011/2019) and Associate Laboratory LSRE-LCM, POCI-01-0145-FEDER-006984 (Ref. FCT UID/EQU/50020/2019), and project MultiBiorefinery (POCI-01-0145-FEDER-016403), all financed by national funds through the FCT/MCTES (PIDDAC) and when appropriate co-financed by FEDER under the PT2020 Partnership Agreement.

\section{Conflicts of interest}

There are no conflicts to declare.

\section{Notes and references}

1 A. P. Abbott, G. Capper, D. L. Davies, R. K. Rasheed and V. Tambyrajah, Chem. Commun., 2003, 70-71.

2 A. P. Abbott, D. Boothby, G. Capper, D. L. Davies and R. K. Rasheed, J. Am. Chem. Soc., 2004, 126, 9142-9147.

3 M. A. R. Martins, S. P. Pinho and J. A. P. Coutinho, J. Solution Chem., 2019, 48(7), 962-982.

4 E. L. Smith, A. P. Abbott and K. S. Ryder, Chem. Rev., 2014, 114, 11060-11082.

5 A. P. Abbott, G. Capper, D. L. Davies, K. J. McKenzie and S. U. Obi, J. Chem. Eng. Data, 2006, 51, 1280-1282.

6 P. Liu, J.-W. Hao, L.-P. Mo and Z.-H. Zhang, RSC Adv., 2015, 5, 48675-48704.

7 S. Riaño, M. Petranikova, B. Onghena, T. Vander Hoogerstraete, D. Banerjee, M. R. S. Foreman, C. Ekberg and K. Binnemans, RSC Adv., 2017, 7, 32100-32113.

8 Q. Zhang, K. De Oliveira Vigier, S. Royer and F. Jérôme, Chem. Soc. Rev., 2012, 41, 7108-7146.

9 M. Ruesgas-Ramón, M. C. Figueroa-Espinoza and E. Durand, J. Agric. Food Chem., 2017, 65, 3591-3601.

10 D. J. G. P. van Osch, L. F. Zubeir, A. van den Bruinhorst, M. A. A. Rocha and M. C. Kroon, Green Chem., 2015, 17, 4518-4521.

11 M. A. R. Martins, E. A. Crespo, P. V. A. Pontes, L. P. Silva, M. Bülow, G. J. Maximo, E. A. C. Batista, C. Held, S. P. Pinho and J. A. P. Coutinho, ACS Sustainable Chem. Eng., 2018, 6, 8836-8846.

12 P. Stott, J. Controlled Release, 1998, 50, 297-308.

13 Y. Kaplun-Frischoff and E. Touitou, J. Pharm. Sci., 1997, 86, 1394-1399.

14 M. Lazerges, I. B. Rietveld, Y. Corvis, R. Céolin and P. Espeau, Thermochim. Acta, 2010, 497, 124-128.

15 D. J. G. P. van Osch, C. H. J. T. Dietz, J. van Spronsen, M. C. Kroon, F. Gallucci, M. van Sint Annaland and R. Tuinier, ACS Sustainable Chem. Eng., 2019, 7, 2933-2942.

16 S. O. L. Potter, A Compend of Materia Medica, Therapeutics, and Prescription Writing, 1902.

17 A. Klamt, J. Phys. Chem., 1995, 99, 2224-2235.

18 A. Klamt, V. Jonas, T. Bürger and J. C. W. Lohrenz, J. Phys. Chem. A, 1998, 102, 5074-5085. 